\title{
Cataclysmic variables as gravitational wave sources
}

\author{
Rosa Poggiani* \\ Università di Pisa and Istituto Nazionale di Fisica Nucleare, Sezione di Pisa \\ E-mail: rosa.poggianiedf.unipi.it
}

\begin{abstract}
The recent direct detection of gravitational waves has triggered the interest in gravitational wave sources. Some sources are potentially observable only with space based interferometers, such as the forthcoming LISA scheduled for launch after 2030. Cataclysmic variables are binary sources whose emission is in the sensititivity band of LISA. The paper presents an estimation of the gravitational wave emission of about five hundreds cataclysmic variables. The most promising sources are AM CVn systems.
\end{abstract}

The Golden Age of Cataclysmic Variables and Related Objects IV 11-16 September, 2017

Palermo, Italy

\footnotetext{
* Speaker.
} 


\section{Introduction}

The direct detection of gravitational waves from the binary black hole merger GW150914 [1] by the two Advanced LIGO interferometers during run $\mathrm{O} 1$ has opened a new observational window in astrophysics. Additional black hole mergers have been detected by Advanced LIGO: GW151226 [2] during run O1, and GW170104 [3], GW170608 [4] during run O2. Advanced Virgo joined O2 in August 2017. The network of Advanced LIGO and Advanced Virgo has detected the binary black hole merger GW170814 [5] and the first coalescence of a binary neutron star system [6]. The signatures of black hole and neutron star mergers in ground based interferometers are chirps spanning the frequency region from a few $\mathrm{Hz}$ to some hundreds $\mathrm{Hz}$. The spectrum of gravitational waves is very extended, from $10^{-10}$ to $10^{4} \mathrm{~Hz}$, and includes a large variety of astronomical sources (Fig. 1) that involve different detection techniques [40].

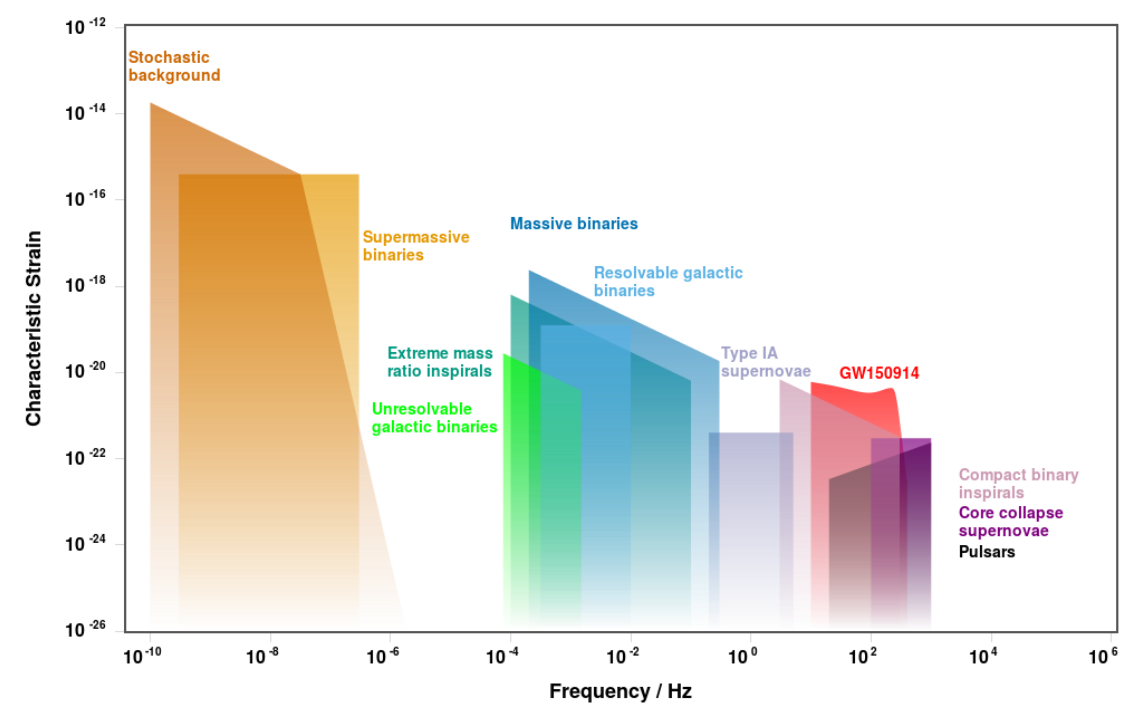

Figure 1: The spectrum of gravitational waves, based on the tool at http://rhcole.com/apps/GWplotter/

The Very Low Frequency region, below $10^{-5} \mathrm{~Hz}$, includes a stochastic background and the radiation of supermassive binaries. The region can be accessed using pulsar timing techniques [41]. The Low Frequency region, between $10^{-5}$ and $0.1 \mathrm{~Hz}$, includes the coalescence of massive binaries and of extreme mass ratio systems and the emission of resolvable and unresolvable galactic binaries; it can be probed by space based interferometers. The High Frequency region, above some $\mathrm{Hz}$, is the domain of ground based interferometers and includes the radiation by the mergers of stellar mass black holes, of neutron stars and of the still undetected neutron star/black hole mergers, core collapse supernovae, pulsars, stochastic background of unresolved black hole and neutron star binaries.

The forthcoming space based laser interferometer LISA aims to detect gravitazional waves in the frequency range from $10^{-5} \mathrm{~Hz}$ to $10^{-1} \mathrm{~Hz}$, a region precluded to ground based instruments by the presence of seismic noise. As mentioned above, the science reach of the low frequency region is large, including the coalescence of supermassive black holes, contact binary systems and binaries including compact objects, such as white dwarfs, neutron stars or black holes. Electromagnetic 
observations of binary systems are often available, providing information about the sky position and the orbital period of the system. Due to the large number of binary systems, an astrophysical background from binary systems is expected, expecially from galactic unresolved contact binaries, pairs of white dwarfs or neutron stars and cataclysmic binaries [32], [19].

The gravitational wave emission of binary systems has been thoroughly investigated, making them the better understood sources [53], [39], [28], [34], [27], [32], [19], [59]. The estimation of gravitational emission of a binary system requires the knowledge of the orbital period, of the masses of the components and of the distance. The cataclysmic variables, binary system where a white dwarf is accreting material from another star [58], are of special interest. To date, more than one thousand systems are known [48], [49]. The total number of galactic cataclysmic variables is estimated to be of the order of $10^{6}$ [32]. Their orbital periods range from minutes to hours, thus their gravitational emission, that occurs at twice the orbital period, is in the LISA frequency range.

In the following, the estimation of the gravitational wave emission of about five hundreds cataclysmic variables is presented. Following the approach by [38], who estimated the emission for 160 systems, I have estimated the gravitational wave emission for systems where all parameters (distance, orbital period and masses) are known, complementing them with the systems where only the distance and the orbital period are known.

The paper firstly presents a summary of the properties of cataclysmic variables relevant for the gravitational wave emission. Then the problem of the distance to cataclysmic systems is discussed. The properties and the sensitivity of the LISA interferometer are presented later. Finally, the gravitational wave emission is estimated for abot five hundred systems, a factor of three larger than previous estimations reported in literatures [18], [38].

\section{Cataclysmic variables: orbital periods and masses}

The cataclysmic variables have been catalogued by Ritter and Kolb [48], [49]; the catalogue is available online ${ }^{1}$. The catalogue version 7.23 contains the main parameters of more than 1400 systems. The orbital period is known for most cataclysmic variables. The period distribution is reported in Fig. 2, that clearly shows the period gap between two and three hours.

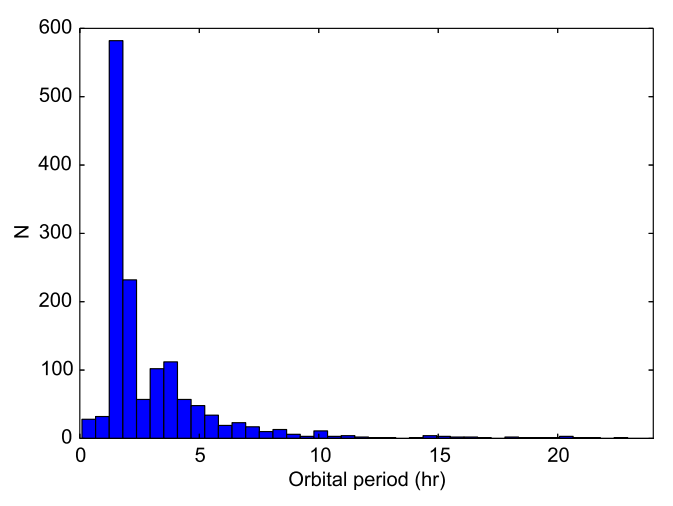

Figure 2: Distribution of the orbital periods of cataclysmic variables

\footnotetext{
${ }^{1}$ http://wwwmpa.mpa-garching.mpg.de/RKcat/
} 
The masses of the primary and secondary stars are known only for a small fraction of all known cataclysmic variables, about 10\%. The distribution of the masses of the primary (about 150 systems) and of secondary stars (about 140 systems) are reported in Fig. 3, left and right.
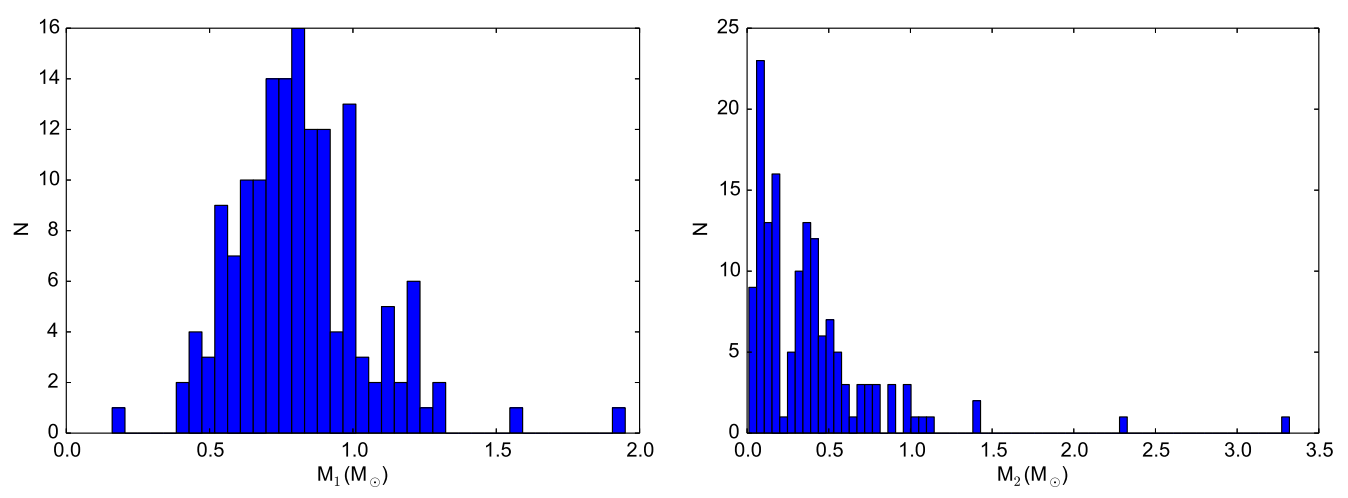

Figure 3: Distribution of the mass of the primary (left) and secondary (right) of cataclysmic variables

I have estimated the masses of the primary and secondary stars with missing values following the approach by [38]. The mass of the primary is estimated the unweigthed average of the systems below the period gap, in the period gap and above the period gap:

$$
\begin{array}{r}
M_{1}=0.76 M_{\odot} \text { below period gap } \\
M_{1}=0.78 M_{\odot} \text { in period gap } \\
M_{1}=0.85 M_{\odot} \text { above period gap }
\end{array}
$$

The mass of the secondary has been estimated with a mass-period relation, following the approach proposed by [52]. I have fitted the mass versus orbital period for 146 systems with periods smaller than ten hours:

$$
M_{2}\left(M_{\odot}\right)=0.0084+0.093 P(h r)
$$

\section{Cataclysmic variables: distances}

The Ritter and Kolb catalogue does not report the distances of the cataclysmic variables. Compilations of distances including a few tens systems have been presented by [21], [44], [45]. I have prepared a compilation of distances, combining the values in the literature that have been obtained using different methods.

The parallax is a primary method for measuring the distance of celestial objects and is used as a reference for other methods. In the following, the parallax based distance will be used when available. Distance based on parallax measurements using ground and space based telescopes are available for about fifty systems. Ground based parallax measurements have been obtained by [54] and [55] for 26 cataclysmic variables. Parallax measurements with HST are available for 19 systems [29], [36], [37], [22], [23], [30], [31], [50]. Nine additional parallaxes have been measured 
with Hipparcos [26]. The first data release of GAIA has provided parallaxes for 16 cataclysmic variables [46].

Limits to the distance of cataclysmic variables can be set by using the infrared $K$ magnitude of the secondary star, as suggested by [16]. The surface brightness is calibrated as a function of effective temperature, relying on the small sensitivity to the evolutionary level of the secondary. The $K$ surface brightness parameter is related to the $V-K$ colour index, a proxy of the effective temperature, via a linear relation or a combination of linear relations in different regions [16]. The surface brightness is also related to the angular diameter of the star, allowing to use compilations of stellar radii (see e.g. [17]). The features of the energy distribution of the cataclysmic variables has been addressed by [56] to determine the separate contributions of the secondary star and of the accretion disk. The origin of the infrared emission in cataclysmic variables has been discussed by [20]. The infrared light is produced by the red dwarf and by the accretion disk, but near infrared photometry cannot disentangle the two contributions, allowing only to set limits to the fraction from the secondary [20]. The method of $K$ surface brightness [16] has been updated with larger datasets by [21] and [47]. Recently, the surface brightness of field dwarfs has been calibrated as a function of $V-K$ color and the spectral type [24]. An approach of distance estimation using theoretical models for the secondary star, that is assumed to fill the Roche lobe, has been presented by [33]. Since the method of infrared magnitude generally does not provide distance values, but only limits, it will not be used in the following.

A new calibration of absolute magnitude of cataclysmic variable based on 2MASS infrared data has been proposed by [7], [8]. The absolute magnitude $M_{J}$ is a function of the orbital period $P$ and of the colours $(J-H)_{0},(H-K)_{0}$, according to the Period-Luminosity-Relation [7], [8]:

$$
M_{J}=-0.894-5.721 \log P(\text { days })+2.598(J-H)_{0}+7.380\left(H-K_{s}\right)_{0}
$$

The approach has produced an homogenous sample of more than four hundreds distances to cataclysmic variables.

The distance of novae can be measured by using different methods. The methods of expansion parallax [25], [51] relies on observations of shells around novae in the years or decades after the outburst. The angular expansion rate is combined with the measurement of the radial velocity [25], [51]. The approach must take into account the non isotropic mass ejection and the inclination of the system [25], [57]. Recently, a new method based on the position of red clump giants on colourmagnitude diagrams and reddening-distance relations towards galactic novae has been proposed by [43], providing the distance to 73 systems. The method has been calibrated against the parallax or expansion parallax [43].

In the following, I will consider distances based on the parallax, the nova expansion parallax, the Period-Luminosity-Colour relation, since they provide distance values and not limits.

\section{The LISA interferometer}

The initial concept of the LISA space based interferometer, a constellation of three spacecraft in heliocentric orbit lagging Earth by 20 degrees, foresaw an arm length of 5 million km [35]. The design of LISA was later reshaped into eLISA, with a shorter arm length [11], [10]. In response 
to ESA call for missions for L3, a new proposal was presented. The new LISA will be based on three arms with six laser links exchanged between three identical spacecrafts in a triangular formation, with a separation of 2.5 million $\mathrm{km}$. The LISA interferometer will monitor the entire sky, measuring both polarisations of the gravitational waves at the same time, with a sensitivity band extending from below $10^{-5} \mathrm{~Hz}$ to above $10^{-1} \mathrm{~Hz}$ [9]. LISA is scheduled for launching in 2034.

The constellation of three spacecrafts forming LISA will orbit the Sun in a triangular configuration, along heliocentric orbits that will avoid the necessity of orbit corrections. The constellation will be centered in the ecliptic plane, trailing the Earth by about 20 degrees. The plane of the triangle will be inclined by 60 degrees with respect to the ecliptic. The three spececrafts will contain free falling test masses and will controlled to be constantly centered on the masses, without applying forces on the masses. The drag free operation will be achieved by embedding the test masses inside an housing that probes the relative position of the masses and the spacecraft and manages the spacecraft thrusters. Each test mass defines the end side for the optical length and acts as a geodesic reference. The drag free configuration will minimize the effects of force gradients. Each test mass will be a $46 \mathrm{~mm}$ cube made of non magnetic Au-Pt alloy, surrounded by the Gravitational Reference Sensor (GRS), operating with capacitive sensing. Each spacecraft will host two units containing a Gravitational Reference Sensor and an embedded free-falling test mass. The standard Michelson interferometer configuration with direct reflection cannot be used in LISA, due to the large distance between the spacecrafts. The lasers will be used in a transponder configuration: a beam is sent from one spacecraft to the other one, where the laser is phase locked to the incoming beam and sends back a replicated beam. The beam received by the original spacecraft is compared with the local laser beam. The procedure will be used in all three arms of LISA, with two transponders in each arm. The virtual armlength interferometer is built during post-processing with the technique of Time-Delay Interferometry (TDI).

LISA Pathfinder (LPF) [12], launched in December 2015, has been the demonstrator of the LISA concept and of key technologies. The test masses were at a distance of $0.38 \mathrm{~m}$. LISA Pathfinder has placed two test masses in free fall, unperturbed by other stray forces at a level more than five times better than the orginal specifications [13], [14].

\section{Gravitational waves from cataclysmic variables}

The gravitational wave emission of cataclysmic variables has been addressed by [18] and by [38], who investigated a sample of about 160 objects each. Cataclysmic variables, as other binary systems, emit gravitational waves at twice the orbital frequency and harmonics. The contribution of the harmonics is generally negligible, since the orbits are progressively circularized during the evolution. The gravitational wave characteristic amplitude produced by a binary system is [53]:

$$
h=8.7 \times 10^{-21}\left(\frac{\mu}{M_{\odot}}\right)\left(\frac{M}{M_{\odot}}\right)^{\frac{2}{3}}\left(\frac{100 p c}{r}\right)\left(\frac{f}{10^{-3} H z}\right)^{\frac{2}{3}}
$$

where $M=M_{1}+M_{2}, \mu=\frac{M_{1} M_{2}}{M_{1}+M_{2}}, M_{1}, M_{2}$ are the masses of the primary and secondary star, $r$ the distance of the cataclysmic and $f$ the gravitational wave frequency. 
The strain $h$ for the sample of about 500 hundred systems investigated in the present paper is shown in Fig. 4. The strain of systems with known masses and of systems with estimated masses are reported with different symbols. The solid curve is the sky averaged sensitivity of the new LISA design reported by [15], with an arm length of $2.5 \times 10^{6} \mathrm{~km}$, an acceleration noise based on the LISA Pathfinder performances and the confusion noise estimated by [9]. For comparison, the dotted curve is the sensitivity of the LISA original design with 5 million $\mathrm{km}$ arm length [35] ${ }^{2}$. The dashed curve is the contribution of the confusion noise, the astrophysical background produced by unresolved binary systems [32], [19], as discussed by [9]. An observation time of 2 years is assumed.

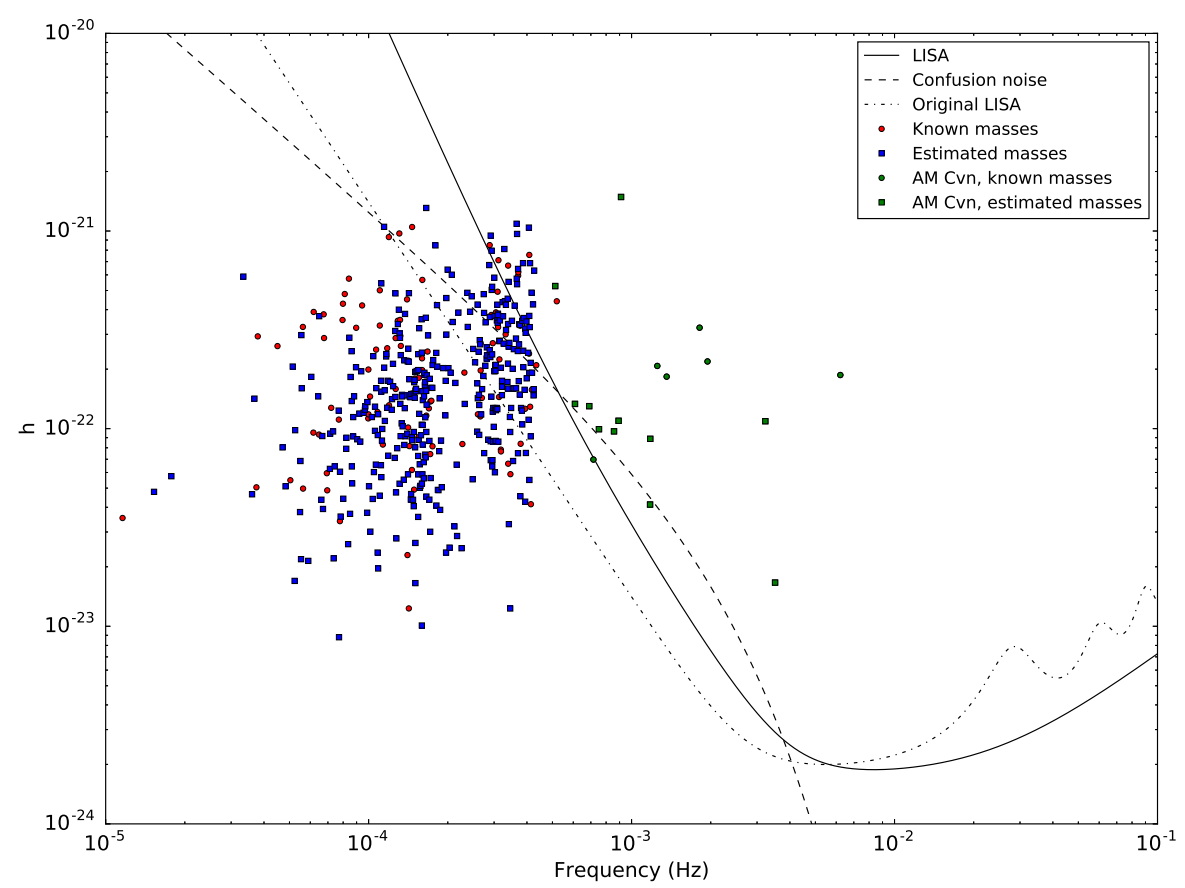

Figure 4: Gravitational wave emission of cataclysmic variables with known masses (red circles) and with estimated masses (blue squares); the AM CVN systems with known masses and estimated masses are marked by green circles and green squares, respectively; the solid curve is the instrumental sensitivity of the new design LISA interferometer [15], the dotted line of the original LISA [35], the dashed line is the binary confusion noise [9]

The majority of potentially detectable systems are AM CVn objects [42] and short period systems in general. The AM CVn objects are marked by green symbols in Fig. 4. The better low frequency sensitivity of the original LISA design is however made uneffective by the confusion noise.

\section{Conclusions}

The present estimation of gravitational wave emission from cataclysmic variables includes about five hundreds systems, a factor three larger than the previous estimations. The strongest

\footnotetext{
${ }^{2} \mathrm{http} / / / \mathrm{www}$. srl.caltech.edu/s̃hane/sensitivity/
} 
sources are AM CVn and short period systems.

\section{References}

[1] B. P. Abbott et al., PRL 116 (2016) 061102.

[2] B. P. Abbott et al., PRL 116 (2016) 241103.

[3] B. P. Abbott et al., PRL 118 (2017) 221101.

[4] B. P. Abbott et al., ApJ 851 (2017) L35.

[5] B. P. Abbott et al., PRL 119 (2017) 141101.

[6] B. P. Abbott et al., PRL 119 (2017) 161101.

[7] T. Ak et al., NewA 12 (2007) 446.

[8] T. Ak et al., NewA 13 (2008) 133.

[9] P. Amaro-Seoane et al., arXiv:1702.00786.

[10] P. Amaro-Seoane et al., CQG 29 (2012) 124016.

[11] P. Amaro-Seoane et al., GW Notes 6 (2013) 4.

[12] F. Antonucci et al., CQG 29 (2012) 124014.

[13] M. Armano et al., PRL 116 (2016) 231101.

[14] M. Armano et al., PRL 120 (2018) 061101.

[15] S. Babak et al., PRD 95 (2017) 103012.

[16] J. Bailey, MNRAS 197 (1981) 31.

[17] T. G. Barnes and D. S. Evans, MNRAS 174 (1996) 489.

[18] F. Barone et al., GRG 24 (1992) 323.

[19] P. L. Bender and D. Hils, CQG 14 (1997) 1439.

[20] G. Berriman et al., MNRAS 217 (1985) 327.

[21] G. Berriman et al., A\&ASS 68 (1987) 41.

[22] K. Beuermann et al., $A \& A 412$ (2003) 821.

[23] K. Beuermann et al., $A \& A 419$ (2004) 291.

[24] K. Beuermann, $A \& A 460$ (2006) 783.

[25] R. A. Downes and H. W. Duerbeck, AJ 120 (2000) 2007.

[26] H. W. Duerebeck, IBVS 4731 (1991).

[27] C. R. Evans et al., ApJ 323 (1987) 129.

[28] R. L. Forward and D. Berman, PRL 18 (1967) 1071.

[29] T. E. Harrison et al., ApJ 515 (1999) L93.

[30] T. E. Harrison et al., $A J 127$ (2004) 460.

[31] T. E. Harrison et al., ApJ 767 (2013) 7. 
[32] D. Hils et al., ApJ 360 (1990) 65.

[33] C. Knigge, MNRAS 372 (2006) 484.

[34] V. M. Lipunov et al., A\&A 176 (1987) L1.

[35] LISA Study Team, 1998, in LISA Pre-Phase A Report. 2nd Edition, Publication MPQ-233 (1998) Max-Plank Institute for Quantum Optics, Garching.

[36] B. E. McArthur et al., ApJ 520 (1999) L59.

[37] B. E. McArthur et al., ApJ 560 (2001) 907.

[38] M. T. Meliani et al., $A \& A 358$ (2000) 417.

[39] V. N. Mironovskii, SvA 9 (1966) 752.

[40] C. J. Moore et al., CQG 32 (2015) 015014.

[41] C. J. Moore et al., CQG 32 (2015) 055004.

[42] G. Nelemans et al., MNRAS 349 (2004) 181.

[43] A. Özdönmez et al., MNRAS 461 (2016) 1177.

[44] J. Patterson, ApJS 54 (1984) 443.

[45] J. Patterson, MNRAS 411 (2011) 2695.

[46] G. Ramsay et al., A\%A 604 (2017) A107.

[47] T. F. Ramseyer, ApJ 425 (1994) 243.

[48] H. Ritter and U. Kolb, $A \& A 404$ (2003) 301.

[49] H. Ritter and U. Kolb, Acta Polytech. CTU Proc. 2 (2015) 21.

[50] G. H. A. Roelofs et al., ApJ 666 (2007) 1174.

[51] A. J. Slavin et al., MNRAS 276 (1995) 353.

[52] D. A. Smith and V. S. Dhillon, MNRAS 301 (1998) 767.

[53] K. S. Thorne, in Three Hundreds Years of Gravitation (1987) 330, Cambridge University Press, Cambridge, eds. S. Hawking and W. Israel.

[54] J. R. Thorstensen, AJ 126 (2003) 3017.

[55] J. R. Thorstensen et al., AJ 136 (2008) 2107.

[56] R. A. Wade, AJ 87 (1982) 1558.

[57] R. A. Wade eta al., PASP 112 (2000) 614.

[58] B. Warner, Cataclysmic Variable Stars (1995), Cambridge University Press, Cambridge

[59] R. F. Webbink et al., ApJ 314 (1987) 653. 